\title{
JHK-band spectro-interferometry of T Cep with the IOTA interferometer
}

Gerd Weigelt, Udo Beckmann, Jean-Philippe Berger, Thomas Bloecker, Michael K. Brewer, et al.

Gerd Weigelt, Udo Beckmann, Jean-Philippe Berger, Thomas Bloecker, Michael K. Brewer, Karl-Heinz Hofmann, Marc G. Lacasse, Victor Malanushenko, Rafael Millan-Gabet, John D. Monnier, Keiichi Ohnaka, Ettore Pedretti, Dieter Schertl, F. Peter Schloerb, Michael Scholz, Wesley A. Traub, Boris Yudin, "JHK-band spectro-interferometry of T Cep with the IOTA interferometer," Proc. SPIE 4838, Interferometry for Optical Astronomy II, (21 February 2003); doi: 10.1117/12.458659

Event: Astronomical Telescopes and Instrumentation, 2002, Waikoloa, Hawai'i, United States 


\title{
JHK-band spectro-interferometry of T Cep with the IOTA interferometer
}

\author{
G. Weigelt ${ }^{a}$, U. Beckmann ${ }^{a}$, J. Berger ${ }^{b}$, T. Blöcker ${ }^{a}$, M.K. Brewer ${ }^{c}$, \\ K.-H. Hofmann ${ }^{a}$, M. Lacasse ${ }^{b}$, V. Malanushenko ${ }^{e}$, R. Millan-Gabet ${ }^{b}$, J. Monnier ${ }^{b}$,

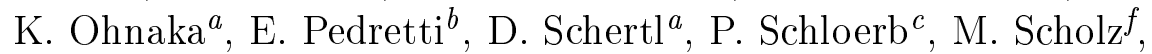 \\ W. Traub ${ }^{b}$, B. Yudin ${ }^{d}$ \\ ${ }^{a}$ Max-Planck-Institut für Radioastronomie, 53121 Bonn, Germany \\ ${ }^{b}$ Harvard-Smithsonian Center for Astrophysics, Cambridge, MA 02138, USA \\ ${ }^{c}$ Physics and Astronomy Dept., Univ. Massachusetts, Amherst, MA 01003, USA \\ ${ }^{d}$ Sternberg Astronomical Institute, 119899 Moscow, Russia \\ ${ }^{e}$ Crimean Astrophysical Observatory, 98409 Crimea, Ukraine \\ fInstitut für Theoretische Astrophysik der Universität Heidelberg, \\ 69121 Heidelberg, Germany, and Institute of Astronomy, \\ School of Physics, University of Sydney NSW 2006, Australia.
}

\begin{abstract}
Our new IOTA ${ }^{1}$ JHK-band beam combiner allows the simultaneous recording of spectrally dispersed J-, H- and K-band Michelson interferograms. In this paper we present our IOTA observations of the Mira star T Cep with this beam combiner (observations in June 2001; four baselines in the range of $14 \mathrm{~m}$ to $27 \mathrm{~m}$ ). The beam combiner optics consists of an anamorphic cylindrical lens system and a prism. From the interferograms of T Cep we derive the visibilities and the $\mathrm{J}_{-}, \mathrm{H}-$, and $\mathrm{K}$-band uniform-disk diameters of $14.0 \pm 0.6$ mas, $13.7 \pm 0.6$ mas and $15.0 \pm 0.6$ mas, respectively. Angular stellar filter radii and Rosseland radii are derived from the measured visibilities by fitting theoretical center-to-limb intensity variations (CLVs) of different Mira star models ${ }^{2,3}$. The available HIPPARCOS parallax $(4.76 \pm 0.75 \mathrm{mas})$ of $\mathrm{T}$ Cep allows us to determine linear radii. For example, from the K-band visibility we derive a Rosseland radius of $329_{-50}^{+70} \mathrm{R}_{\odot}$ if we use the CLVs of the M-models ${ }^{3}$ as fit functions. This radius is in good agreement with the theoretical M-model Rosseland radius of $315 \mathrm{R}_{\odot}$. The comparison of measured stellar parameters (e.g. diameters, effective temperature, visibility shape) with theoretical parameters indicates whether any of the models is a fair representation of $\mathrm{T}$ Cep. The ratios of visibilities of different spectral channels can be measured with higher precision than absolute visibilities. Therefore, we use the visibility ratios $\mathrm{V}\left(\lambda_{1}\right) / \mathrm{V}\left(\lambda_{2}\right)$ to investigate the wavelength dependence of the stellar diameter. We find that the $2.03 \mu \mathrm{m}$ uniform-disk diameter of T Cep is $\sim 1.26$ times larger than the $2.26 \mu \mathrm{m}$ uniform-disk diameter.
\end{abstract}

Keywords: interferometry, Mira stars, near-infrared

\section{INTRODUCTION}

Our new JHK beam combiner built at the MPI for Radioastronomy consists of an anamorphic lens system and a prism. This NIR beam combiner is similar to the dispersed-fringe instruments used at the I2T/GI2T interferometer in the optical wavelength range ${ }^{4}$. The anamorphic lens system is required to obtain a high magnification in the direction perpendicular to the fringe direction and a lower magnification in the direction parallel to the fringes. Figure 1 shows examples of our T Cep interferograms (see also the movie on our home page, http://www.mpifr-bonn.mpg.de/div/ir-interferometry). 

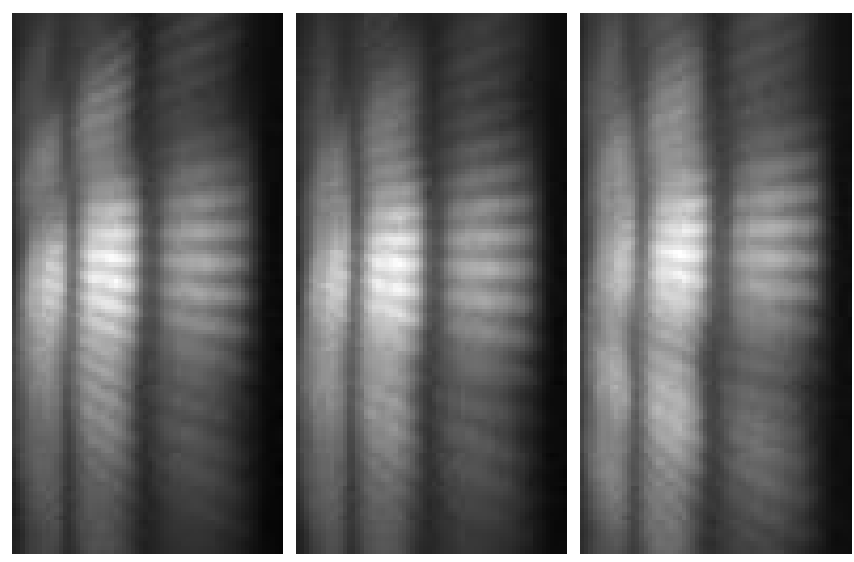

Figure 1. Three consecutive JHK-band IOTA interferograms of T Cep (wavelength range 1-2.3 $\mu \mathrm{m}$ from left to right in each panel)
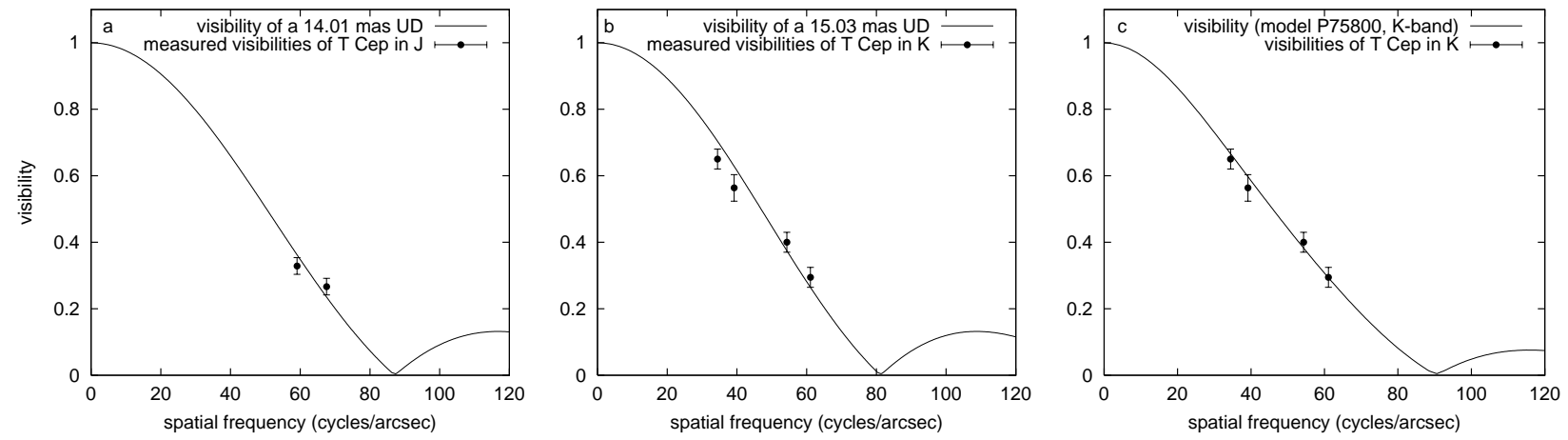

Figure 2. a,b: uniform-disk (UD) fits to the visibilities of T Cep measured in the J- and K-band. c: fit of the model CLV of the best-fitting fundamental mode model P75800 to the measured K-band visibilities of $\mathrm{T}$ Cep.

\section{T CEP OBSERVATIONS}

The first observations with the new JHK beam combiner mounted to the IOTA interferometer were carried out on June 6, 9, 10 and 11, 2001. The raw visibilities of each spectral channel and each baseline were derived by evaluating the power spectra of the interferograms (determination of the ratio of off-axis and central peak). The calibrated visibilities were reconstructed from the raw visibilities of the object and the raw visibilities of reference stars. Figures 2a,b show the calibrated $\mathrm{J}$ and $\mathrm{K}$ visibilities and the best-fitting $\mathrm{J}$ and $\mathrm{K}$ uniform-disk (UD) visibility functions. From the UD fits, stellar diameters of $14.0 \pm 0.6$ mas (J-band), $13.7 \pm 0.6$ mas (Hband), and $15.0 \pm 0.6$ mas (K-band) were obtained (along position angle $\sim 0^{\circ}$ ). Figure $2 \mathrm{c}$ shows the fit of the model CLV of the best-fitting fundamental mode model $\mathrm{P} 75800^{3}$ to the observed K-band visibilities of $\mathrm{T}$ Cep (see discussion in Sect. 3).

\section{COMPARISON OF THE OBSERVATIONS WITH MIRA STAR MODELS}

We derive angular diameters from the measured visibilities by fitting different theoretical center-to-limb intensity variations of different Mira star models (Bessel, Scholz \& Wood ${ }^{2}$; Hofmann, Scholz \& Wood ${ }^{3}$ ). These models were developed as possible representations of the prototype Mira variable o Ceti, and hence have periods $P$ very close to the 332 day period of o Ceti (the period of T Cep is 382 days); they differ in pulsation mode, as well as in modelling assumptions, assumed mass $M$ and luminosity $L$. The five model series represent stars pulsating in the fundamental mode $(f ; \mathrm{D}, \mathrm{P}$ and $\mathrm{M})$ or in the first-overtone mode $(o ; \mathrm{E}$ and $\mathrm{O})$. Table 1 lists these model series $\left(R_{\mathrm{p}}=\right.$ Rosseland radius of the non-pulsating "parent" star of the Mira variable ${ }^{2,3} ; T_{\text {eff }}=$ 
effective temperature). In the following sections we compare predictions of these models at different phases and cycles with our measurements.

Table 1. Mira model series (see text)

\begin{tabular}{|c|c|c|c|c|c|c|}
\hline Series & Mode & $P(\mathrm{~d})$ & $M / M_{\odot}$ & $L / L \odot$ & $R_{\mathrm{p}} / R_{\odot}$ & $T_{\text {eff }} / \mathrm{K}$ \\
\hline $\mathrm{D}$ & $\mathrm{f}$ & 330 & 1.0 & 3470 & 236 & 2900 \\
$\mathrm{E}$ & $\mathrm{o}$ & 328 & 1.0 & 6310 & 366 & 2700 \\
$\mathrm{P}$ & $\mathrm{f}$ & 332 & 1.0 & 3470 & 241 & 2860 \\
$\mathrm{M}$ & $\mathrm{f}$ & 332 & 1.2 & 3470 & 260 & 2750 \\
$\mathrm{O}$ & $\mathrm{o}$ & 320 & 2.0 & 5830 & 503 & 2250 \\
\hline
\end{tabular}

\subsection{Monochromatic radius $R_{\lambda}$, Rosseland radius $R$ and stellar filter radius $R_{\mathrm{f}}$}

Monochromatic radius $R_{\lambda}$ : We use the conventional radius definition where the monochromatic radius $R_{\lambda}$ is given by the distance from the star's center at which the optical depth equals unity $\left(\tau_{\lambda}=1\right)$.

Rosseland radius $R$ : In analogy, the photospheric stellar radius or Rosseland radius $R$ is given by the distance from the star's center at which the Rosseland optical depth equals unity $\left(\tau_{\text {Ross }}=1\right)$.

Stellar filter radius $R_{\mathrm{f}}$ : The stellar radius for filter transmission $\mathrm{f}_{\lambda}$ is the intensity and filter weighted radius $R_{\mathrm{f}}=\int R_{\lambda} I_{\lambda} \mathrm{f}_{\lambda} d \lambda / \int I_{\lambda} \mathrm{f}_{\lambda} d \lambda$, which we call stellar filter radius $R_{\mathrm{f}}$ (see definition in Ref. 6). $R_{\lambda}$ denotes the above monochromatic $\tau_{\lambda}=1$ radius, $I_{\lambda}$ the central intensity spectrum and $f_{\lambda}$ the transmission of the filter. If a spectrograph is used instead of an interference filter, then $f_{\lambda}$ describes the wavelength region which is selected from the spectrally dispersed interferograms.

\subsection{Derived angular stellar filter radii, angular Rosseland radius and linear radii}

The derived angular stellar filter radii $R_{\mathrm{K}, \mathrm{m}}^{a}, R_{\mathrm{H}, \mathrm{m}}^{a}$ and $R_{\mathrm{J}, \mathrm{m}}^{a}$ (corresponding to the model-phase combinations $\mathrm{m})$ were determined by least-squares fits of the model ${ }^{2,3} \mathrm{CLV}$ visibilities to the measured visibilities.

Additionally, the angular Rosseland radii $R_{\mathrm{m}}^{a}$ were derived from the obtained stellar filter radii $R_{\mathrm{K}, \mathrm{m}}^{a}, R_{\mathrm{H}, \mathrm{m}}^{a}$ and $R_{\mathrm{J}, \mathrm{m}}^{a}$ and the theoretical ratios (see Table 3 in Ref. 5 ) between $R / R_{\mathrm{p}}$ and $R_{\mathrm{K}} / R_{\mathrm{p}}, R_{\mathrm{H}} / R_{\mathrm{p}}$, and $R_{\mathrm{J}} / R_{\mathrm{p}}$ predicted by the above Mira star models.

We derived linear stellar filter radii $R_{\mathrm{K}, \mathrm{m}}, R_{\mathrm{H}, \mathrm{m}}, R_{\mathrm{J}, \mathrm{m}}$, and linear Rosseland radii $R_{\mathrm{m}}$ of $\mathrm{T}$ Cep from the angular stellar filter radii $R_{\mathrm{K}, \mathrm{m}}^{a}, R_{\mathrm{H}, \mathrm{m}}^{a}, R_{\mathrm{J}, \mathrm{m}}^{a}$, and Rosseland radii $R_{\mathrm{m}}^{a}$ by using the T Cep HIPPARCOS parallax of $4.76 \pm 0.75$ mas $^{7,8}$.

Fig. 3 shows the linear Rosseland radii $R_{\mathrm{m}}$ derived from the K-band visibilities and also the stellar filter radii $R_{\mathrm{K}, \mathrm{m}}$ for all model-phase combinations $\mathrm{m}$. A comparison of the radii derived from observations and theoretical radii shows that the theoretical Rosseland radii of the fundamental mode D model are too small, and the theoretical Rosseland radii of the first-overtone models $\mathrm{E}$ and $\mathrm{O}$ are too large. However, the theoretical Rosseland radii of the fundamental mode $\mathrm{M}$ and $\mathrm{P}$ model series are at nearly all available near-maximum phases close to the Rosseland radii of $\mathrm{T}$ Cep derived from the observations (for all cycles of the $\mathrm{M}$ model and for 2 of 4 cycles of the $\mathrm{P}$ model). For example, we can compare the theoretical M-model Rosseland radius with the derived phase-averaged linear Rosseland radius obtained from the K-band visibilities and the M-model CLVs. The phase-averaged derived linear Rosseland radius is the average of the Rosseland radii derived with all nearmaximum (i.e. close to our observation) M-models (average over the m-values 17 and 19). The comparison shows that there is very good agreement between the measured uniform disk radius of $337_{-52}^{+72} R_{\odot}$, the derived M-model Rosseland radius of $335_{-55}^{+75} R_{\odot}$ and the theoretical M-model Rosseland radius of $315 \mathrm{R}_{\odot}$. 

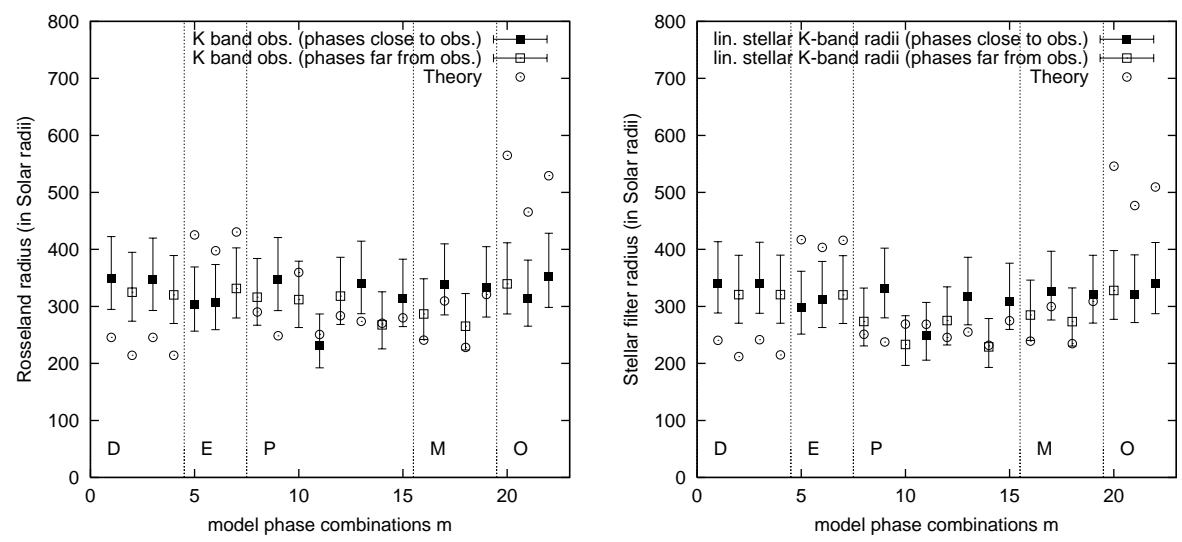

Figure 3. Comparison of $\mathrm{T}$ Cep radii derived from the observations with theoretical model radii. Left: linear Rosseland radii $R_{\mathrm{m}}$ for all 22 model-phase combinations $\mathrm{m}$ (different values of $\mathrm{m}$ correspond to different phases or cycles; see Ref. 5). Right: linear stellar K-band radii for all 22 model-phase combinations $\mathrm{m}$. Filled squares correspond to linear radii derived with CLVs of models with phases close to the phase of our observation, open squares correspond to phases far from our observation (shown for comparison). The theoretical model radii are plotted with open circles.

\section{DIAMETER RATIOS $\frac{\mathrm{D}\left(\lambda_{1}\right)}{\mathrm{D}\left(\lambda_{2}\right)}$ DERIVED FROM VISIBILITY RATIOS $\frac{\mathrm{V}\left(\lambda_{1}\right)}{\mathrm{V}\left(\lambda_{2}\right)}$}

Our JHK interferograms allow us to derive from each interferogram the visibilities of many different spectral channels in the wavelength range of $1-2.3 \mu \mathrm{m}$. We use these visibilities to determine the diameter ratios $\mathrm{D}\left(\lambda_{1}\right) / \mathrm{D}\left(\lambda_{2}\right)$ and compare these observed ratios with theoretical model ratios ${ }^{10}$.

For this goal we have divided our K-band interferograms into three wavelength bins of approximately $0.1 \mu \mathrm{m}$ width and derived the visibility ratios $\mathrm{V}_{2.03 \mu \mathrm{m}} / \mathrm{V}_{2.26 \mu \mathrm{m}}$ and $\mathrm{V}_{2.15 \mu \mathrm{m}} / \mathrm{V}_{2.26 \mu \mathrm{m}}$. From these visibility ratios we can derive diameter ratios which show that the diameter of T Cep is much larger at $2.03 \mu \mathrm{m}$ than at $2.15 \mu \mathrm{m}$ or $2.26 \mu \mathrm{m}$. If uniform-disk model visibilities are fitted to the $\mathrm{T}$ Cep visibilities $\mathrm{V}_{2.03 \mu \mathrm{m}}, \mathrm{V}_{2.15 \mu \mathrm{m}}$, and $\mathrm{V}_{2.26 \mu \mathrm{m}}$ of the $17 \mathrm{~m}$ baseline data, we obtain the diameter ratios $\mathrm{D}_{2.03 \mu \mathrm{m}} / \mathrm{D}_{2.26 \mu \mathrm{m}}=1.26$ and $\mathrm{D}_{2.15 \mu \mathrm{m}} / \mathrm{D}_{2.26 \mu \mathrm{m}}=$ 1.08. These diameter ratios are in good agreement with both the predicted theoretical diameter $\operatorname{ratios}^{10}$ and observations presented in Ref. 11. Further work including a comparison of our J- and H-band observations with Mira models, the wavelength dependence of the diameter in the $\mathrm{H}$ - and J-band, and the derivation of the effective temperature from the measured JHK radii and from coeval $U B V J H K L M$ (June 09, 2001) photometry is reported in Ref. 9.

\section{REFERENCES}

1. W.A. Traub, SPIE $3350,848,1998$

2. M.S. Bessell, M. Scholz, M., and P.R. Wood 1996, A\&A, 307, 481

3. K.-H. Hofmann, M. Scholz, and P.R. Wood 1998, A\&\&A, 339, 846

4. A. Labeyrie, G. Schumacher, M. Dugue, at al. 1986, $A \mathscr{B} A$, 162, 359

5. K.-H. Hofmann, U. Beckmann, T. Blöcker, et al. 2001, New Astronomy, 7, 9-20

6. M. Scholz and Y. Takeda 1987, A\& A, 186, 200 (erratum: 196, 342)

7. ESA 1997, The Hipparcos \& Tycho Catalog, ESA SP-1200, Noordwijk: ESA

8. F. Van Leeuwen, M.W. Feast, P.A. Whitelock, and B. Yudin 1997, MNRAS, 287, 955

9. G. Weigelt, U. Beckmann, T. Blöcker, et al. "Spectro-interferometry of the Mira star T Cep with the IOTA interferometer and comparison with models", in preparation

10. A.P. Jacob and M. Scholz "Effects of molecular contamination of IR near-continuum bandpasses on measurements of M-type Mira diameters", MNRAS, in press

11. R.R. Thompson, M.J. Creech-Eakman and G.T. van Belle "Multi-epoch interferometric study of Mira variables I. Narrowband diameters of RZ Peg and S Laci", ApJ, in press 